\title{
Short Communication Sleep duration and the risk of prostate cancer: the Ohsaki Cohort Study
}

\author{
M Kakizaki,', ', K Inoue', S Kuriyama', T Sone', K Matsuda-Ohmori', N Nakaya', S Fukudo ${ }^{2}$ and I Tsuji' \\ 'Division of Epidemiology, Department of Public Health and Forensic Medicine, Tohoku University Graduate School of Medicine, Sendai, Japan; ${ }^{2}$ Division \\ of Behavioral Medicine, Department of Functional Medical Science, Tohoku University Graduate School of Medicine, Sendai, Japan
}

In a prospective study of prostate cancer incidence ( 127 cases), among 22320 Japanese men, sleep duration was associated with lower risk; the multivariate hazard ratio of men who slept $\geqslant 9 \mathrm{~h}$ per day compared with those who slept less was 0.48 ( $95 \%$ confidence interval: $0.29-0.79, P$ for trend $=0.02$ ).

British Journal of Cancer (2008) 99, I76- 178. doi:10.1038/sj.bjc.6604425 www.bjcancer.com

Published online 10 June 2008

(C) 2008 Cancer Research UK

Keywords: sleep duration; prostate cancer; incidence; Japanese; prospective cohort study

Sleep duration is associated with various health outcomes (Youngstedt and Kripke, 2004) including three observational studies of sleep duration and breast cancer (Verkasalo et al, 2005; McElroy et al, 2006; Pinheiro et al, 2006). Melatonin, which is secreted mainly from the pineal gland and plays a role in sleep duration, is suggested as one of the candidates responsible for the association in breast cancer (Brzezinski, 1997; Schernhammer and Schulmeister, 2004) as it influences the synthesis and secretion of sex hormones by promoting the release of gonadotropin-releasing hormone (Martin and Klein, 1976; Aleandri et al, 1996).

In relation to melatonin, there have been several observational studies of night work, shift work or visual impairment, and sex hormone-related cancers such as prostate or breast (Feychting et al, 1998; Verkasalo et al, 1999; Kliukiene et al, 2001; Megdal et al, 2005; Kubo et al, 2006; Conlon et al, 2007; Schwartzbaum et al, 2007). However, there has been no study of sleep duration and prostate cancer risk, even though prostate cancer, like breast cancer, is also a sex hormone-related cancer.

We therefore examined the association between sleep duration and prostate cancer risk in a population of Japanese men, in whom the mortality of prostate cancer is increasing (Statistics and Information Department, Minister's Secretariat, Ministry of Health Labour and Welfare of Japan, 2007).

\section{MATERIALS AND METHODS}

Details of the Ohsaki National Health Insurance (NHI) Cohort Study have been described previously (Tsuji et al, 1998; Kuriyama et al, 2006). Briefly, this prospective cohort study was started in 1994 and included 26481 men aged $40-79$ years living in the 14 municipalities of Miyagi Prefecture, northeastern Japan. The

*Correspondence: M Kakizaki, Division of Epidemiology, Department of Public Health and Forensic Medicine, Tohoku University Graduate School of Medicine, 2-I Seiryo-machi, Aoba-ku, Sendai, Miyagi, 980-8575, Japan; E-mail: m-kaki@umin.ac.jp

Received 16 March 2008; revised 18 April 2008; accepted 24 April 2008; published online 10 June 2008 response rate was $94.0 \%(N=24895)$ for the questionnaire that was self-administered and included items about sleep duration and other health-related lifestyle factors. The study protocol was reviewed and approved by the ethics committee of Tohoku University School of Medicine.

After exclusion of subjects who had withdrawn from the NHI before follow-up, those who had a history of cancer, those who had omitted responses for sleep duration, and those who had reported sleep duration of less than $4 \mathrm{~h}$ or more than $12 \mathrm{~h}, 22320$ subjects remained. To follow-up participants for mortality and migration, we reviewed the NHI withdrawal history files for 1995-2001. Through the Miyagi Prefectural Cancer Registry, we identified 127 incident cases of prostate cancer. During the study period, there had been no mass screening programme for prostate cancer in this area. Clinical stage was classified according to the TNM system as localised (T1-T2), advanced (T3-T4), metastatic $(\mathrm{N}+$ and/or $\mathrm{M}+$ ), or unknown.

With regard to the sleep duration, participants answered the mean integer number of hours of sleep per day during the last year. Because of the small number who slept for less than $7 \mathrm{~h}$ and more than $8 \mathrm{~h}$, we categorised sleep duration into three groups: $\leqslant 6,7-8$, and $\geqslant 9 \mathrm{~h}$ per day. We estimated hazard ratios (HRs) and $95 \%$ confidence intervals (CIs) of prostate cancer incidence according to sleep duration, using the Cox proportional hazards model, with adjustment for age and potential confounders. The continuous $P$ for trend was calculated by treating sleep duration as a continuous variable, and the categorical $P$ for trend by treating each category as a continuous variable. Interactions between the risk and all confounders were tested through the addition of crossproduct terms to multivariate model.

All statistical analyses were performed using SAS statistical software, version 9.1 (SAS Institute Inc, Cary, NC, USA), and all those reported were two-sided; differences at $P$-values of $<0.05$ were accepted as significant.

\section{RESULTS}

Table 1 shows the baseline characteristics of subjects according to sleep duration. Subjects who slept $9 \mathrm{~h}$ or more per day (long 
sleepers) were older, less likely to be employed and married, and more likely to have a history of disease. Those who slept $6 \mathrm{~h}$ or less per day (short sleepers) were more likely to have never smoked and less likely to walk more than $1 \mathrm{~h}$ per day.

We found an inverse association between sleep duration and risk. The HR of prostate cancer in short sleepers was $1.34(95 \%$ CI: $0.83-2.17)$ and the $\mathrm{HR}$ for long sleepers was 0.48 (95\% CI: $0.29-0.79)$ ( $P$ for trend $=0.02$ ). This result did not change substantially when subjects whose event occurred within 3 years of baseline $(N=46)$ were excluded and analysis of each clinical stage (localised or advanced/metastatic) was examined (Table 2).

In addition, we examined in detail, confounding and effect modification by age and other covariates on the associations with sleep duration. No significant interaction was observed between sleep duration and other confounding factors for risk on a multiplicative scale (data not shown).

\section{DISCUSSION}

In the first study to address the question, we found an inverse association between sleep duration and the risk of prostate cancer in Japanese men. There have, however, been three observational

Table I Baseline characteristics of the subjects according to sleep duration

\begin{tabular}{lccc}
\hline Sleep duration & $\leqslant \mathbf{6}$ & $\mathbf{7 - 8}$ & $\geqslant \mathbf{9}$ \\
\hline Number of subjects & $267 \mid$ & 15 I27 & 4522 \\
Mean age, s.d. (years) & $57.7($ I I.0) & $58.0(10.4)$ & $64.0(9.3)$ \\
Mean body mass index, s.d. $\left(\mathrm{kg} \mathrm{m}^{-2}\right)$ & $23.7(3.2)$ & $23.4(2.9)$ & $23.1(3.4)$ \\
Having history of diseases $^{\mathrm{a}}(\%)$ & 31.3 & 29.4 & 38.9 \\
Employed (\%) & 59.2 & 61.0 & 47.8 \\
Married (\%) & 80.1 & 82.2 & 77.0 \\
Never smoked (\%) & 19.4 & 18.2 & 15.9 \\
Never drank (\%) & 15.5 & 16.3 & 14.8 \\
Walking I h per day or more (\%) & 42.6 & 45.7 & 43.4 \\
Having family history of cancer (\%) & 30.0 & 29.3 & 28.4 \\
\hline
\end{tabular}

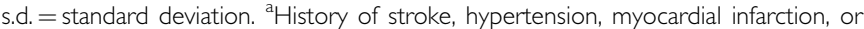
diabetes mellitus. studies of sleep duration and breast cancer risk (McElroy et al, 2006; Pinheiro et al, 2006). Among these, one reported a decreased risk in long sleepers (Verkasalo et al, 2005), the second reported no association (Pinheiro et al, 2006), whereas the third reported an increased risk in long sleepers (McElroy et al, 2006). Among possible reasons for these differences from our findings (apart from the site of cancer), McElroy et al (2006) conducted a casecontrol study while Pinheiro et al (2006) studied residential nurses in the United States with rotating shift work and varying timing of sleep so that generalising from their results may be inappropriate.

Melatonin may be a factor in these inverse associations with sex hormone-related cancers (Brzezinski, 1997; Schernhammer and Schulmeister, 2004; Shiu, 2007). A decreased sleep duration results in a shorter duration of nocturnal melatonin secretion (Wehr, 1991). Melatonin may have an inhibitory effect on gonadal function, including the synthesis and secretion of sex hormones, by promoting the release of gonadotropin-releasing hormone (Martin and Klein, 1976; Aleandri et al, 1996). It also exerts an antiproliferative effect on prostate and breast cancer cell lines (Shiu, 2007).

Strengths of the present study include its prospective nature and its base in the general population. In addition, the Miyagi Prefectural Cancer Registry is one of the earliest and most accurate population-based cancer registries in Japan (Takano and Okuno, 1997); so our data on cancer incidence are considered sufficiently reliable.

Methodological limitations include the fact that sleep duration was self-reported. Second, we had no information about sleep quality, the timing of sleep, the use of sleep medication, the presence of sleeping disorders or rotating shift work. Such factors influence sleep duration and thereby might affect the findings.

In conclusion, we have found a significant inverse association between sleep duration and the risk of prostate cancer incidence in Japanese men.

\section{ACKNOWLEDGEMENTS}

This study was supported by a grant-in-aid for Cancer Research and for the Third Term Comprehensive Ten-Year Strategy for Cancer Control (H18-3jigan-ippan-001), Ministry of Health, Labour and Welfare, Japan.

Table 2 Cox proportional hazard ratios (HRs) for prostate cancer incidence by sleep duration in Japanese men

\begin{tabular}{|c|c|c|c|c|c|}
\hline & \multicolumn{3}{|c|}{ Sleep duration (hours/ per day) } & \multirow[b]{2}{*}{$P$ for trend ${ }^{a}$} & \multirow[b]{2}{*}{$P$ for trend ${ }^{b}$} \\
\hline & $\leqslant 6$ & $7-8$ & $\geqslant 9$ & & \\
\hline Person-years & 16716 & 94786 & 27104 & & \\
\hline \multicolumn{6}{|l|}{ All prostate cancer } \\
\hline Number of cases & 21 & 87 & 19 & & \\
\hline Age-adjusted HR (95\% Cl) & $1.34(0.83-2.15)$ & 1.00 (reference) & $0.46(0.28-0.75)$ & 0.01 & 0.0003 \\
\hline Multivariate HRI $(95 \% \mathrm{Cl})^{c}$ & $1.34(0.83-2.17)$ & 1.00 (reference) & $0.48(0.29-0.79)$ & 0.02 & 0.0007 \\
\hline Multivariate HR2 $(95 \% \mathrm{Cl})^{d}$ & $1.38(0.77-2.48)$ & 1.00 (reference) & $0.36(0.18-0.72)$ & 0.01 & 0.0009 \\
\hline \multicolumn{6}{|l|}{ Localised prostate cancer } \\
\hline Number of cases & 4 & 19 & 3 & & \\
\hline Multivariate $\mathrm{HRI}(95 \% \mathrm{Cl})^{c}$ & $1.13(0.38-3.35)$ & 1.00 (reference) & $0.29(0.09-0.997)$ & 0.11 & 0.05 \\
\hline \multicolumn{6}{|c|}{ Advanced or metastatic prostate cancer } \\
\hline Number of cases & 8 & 25 & 8 & & \\
\hline Multivariate HRI $(95 \% \mathrm{Cl})^{c}$ & $1.82(0.82-4.05)$ & I.00 (reference) & $0.79(0.35-1.77)$ & 0.30 & 0.12 \\
\hline
\end{tabular}

$\mathrm{Cl}=$ confidence interval. ${ }^{a} P$ for trend values were calculated by treating sleep duration as a continuous variable. ${ }^{b} p$ for trend values were calculated by treating each category of sleep duration as a continuous variable. 'Multivariate HRI was adjusted for age ( $<45,45-49,50-54,55-59,60-64,65-69,70-74$, and 75+ years old); marital status (married or unmarried); education (junior high school or less, high school, college/university or higher); job status (employed or unemployed); history of diseases (history of stroke, hypertension, myocardial infarction, or diabetes mellitus); family history of cancer (presence or absence in first-degree relatives); body mass index ( $<$ I8.5, I8.5-24.9, or $\geqslant 25.0 \mathrm{~kg} \mathrm{~m}^{-2}$ ); cigarette smoking (never smoked, smoked in the past, currently smoking $1-19$ cigarettes per day, or currently smoking $\geqslant 20$ cigarettes per day); alcohol consumption (never drank alcohol, drank in the past, or currently drinking); walking status ( $<\mathrm{I}$ h per day or $\geqslant 1 \mathrm{~h}$ per day). ${ }^{\mathrm{d}}$ Multivariate $\mathrm{HR} 2 \mathrm{was}$ estimated excluding 46 subjects who were diagnosed with prostate cancer within the first 3 years from baseline and was adjusted for using the same variables as in multivariable HRI. 


\section{REFERENCES}

Aleandri V, Spina V, Morini A (1996) The pineal gland and reproduction. Hum Reprod Update 2: 225-235

Brzezinski A (1997) Melatonin in humans. N Engl J Med 336: 186-195

Conlon M, Lightfoot N, Kreiger N (2007) Rotating shift work and risk of prostate cancer. Epidemiology 18: $182-183$

Feychting M, Osterlund B, Ahlbom A (1998) Reduced cancer incidence among the blind. Epidemiology 9: $490-494$

Kliukiene J, Tynes T, Andersen A (2001) Risk of breast cancer among Norwegian women with visual impairment. $\mathrm{Br}$ J Cancer 84 $397-399$

Kubo T, Ozasa K, Mikami K, Wakai K, Fujino Y, Watanabe Y, Miki T, Nakao M, Hayashi K, Suzuki K, Mori M, Washio M, Sakauchi F, Ito Y, Yoshimura T, Tamakoshi A (2006) Prospective cohort study of the risk of prostate cancer among rotating-shift workers: findings from the Japan collaborative cohort study. Am J Epidemiol 164: 549-555

Kuriyama S, Shimazu T, Ohmori K, Kikuchi N, Nakaya N, Nishino Y, Tsubono Y, Tsuji I (2006) Green tea consumption and mortality due to cardiovascular disease, cancer, and all causes in Japan: the Ohsaki study. JAMA 296: $1255-1265$

Martin JE, Klein DC (1976) Melatonin inhibition of the neonatal pituitary response to luteinizing hormone-releasing factor. Science 191: $301-302$

McElroy JA, Newcomb PA, Titus-Ernstoff L, Trentham-Dietz A, Hampton JM, Egan KM (2006) Duration of sleep and breast cancer risk in a large population-based case-control study. J Sleep Res 15: 241-249

Megdal SP, Kroenke CH, Laden F, Pukkala E, Schernhammer ES (2005) Night work and breast cancer risk: a systematic review and metaanalysis. Eur J Cancer 41: 2023-2032

Pinheiro SP, Schernhammer ES, Tworoger SS, Michels KB (2006) A prospective study on habitual duration of sleep and incidence of breast cancer in a large cohort of women. Cancer Res 66: 5521-5525
Schernhammer ES, Schulmeister K (2004) Melatonin and cancer risk: does light at night compromise physiologic cancer protection by lowering serum melatonin levels? Br J Cancer 90: 941 - 943

Schwartzbaum J, Ahlbom A, Feychting M (2007) Cohort study of cancer risk among male and female shift workers. Scand J Work Environ Health 33: 336-343

Shiu SY (2007) Towards rational and evidence-based use of melatonin in prostate cancer prevention and treatment. J Pineal Res 43: 1 -9

Statistics and Information Department, Minister's Secretariat, Ministry of Health Labour and Welfare of Japan (2007) Vital Statistics of Japan, 2005. Tokyo (in Japanese): Health and Welfare Statistics Association

Takano A, Okuno Y (1997) Japan, Miyagi Prefecture. In: International Agency for Research on Cancer Parkin D, Whelan S, Ferlay J, Raymond L,Young J (eds), Vol. 17, pp 386-389. Lyon: IARC

Tsuji I, Nishino Y, Ohkubo T, Kuwahara A, Ogawa K, Watanabe Y, Tsubono Y, Bando T, Kanemura S, Izumi Y, Sasaki A, Fukao A, Nishikori M, Hisamichi S (1998) A prospective cohort study on National Health Insurance beneficiaries in Ohsaki, Miyagi Prefecture, Japan: study design, profiles of the subjects and medical cost during the first year. J Epidemiol 8: 258-263

Verkasalo PK, Lillberg K, Stevens RG, Hublin C, Partinen M, Koskenvuo M, Kaprio J (2005) Sleep duration and breast cancer: a prospective cohort study. Cancer Res 65: 9595-9600

Verkasalo PK, Pukkala E, Stevens RG, Ojamo M, Rudanko SL (1999) Inverse association between breast cancer incidence and degree of visual impairment in Finland. Br J Cancer 80: 1459-1460

Wehr TA (1991) The durations of human melatonin secretion and sleep respond to changes in daylength (photoperiod). J Clin Endocrinol Metab 73: $1276-1280$

Youngstedt SD, Kripke DF (2004) Long sleep and mortality: rationale for sleep restriction. Sleep Med Rev 8: 159-174 